\title{
DEVELOPMENTS IN THE MOTIONAL NARROWING OF THE NMR SPECTRA OF SOLIDS- MICROSCOPIC AND MACROSCOPIC
}

\author{
E. R. ANDREW \\ Department of Physics, University of Nottingham, \\ Nottingham, England
}

\begin{abstract}
An investigation of microscopic motional narrowing of $\mathrm{nmr}$ spectra of polycrystalline tetra-, tri-, di- and mono-methylammonium chloride is reported. A diversity of rotational motions of the ions and of the methyl groups within the ions is exhibited. The problem of detailed interpretation of the line-narrowing is particularly acute for two of these compounds, and also for solid l-valine.

The proper use of the decreasing second moment of motionally-narrowed $n m r$ spectra as an alternative means of obtaining an activation energy is discussed. The variation of second moment with correlation time has been calculated on the basis of Kubo and Tomita's theory of motional narrowing, and the use of the measured second moments to extract correlation times is described.

Conformational motion in solids and the consequent narrowing of their $\mathrm{nmr}$ spectra is discussed. Consideration is given to the possibility of a conformational order-disorder process, with special reference to solid cyclobutane. The variation of $\mathrm{nmr}$ linewidth with order parameter has been calculated. The propagation of conformational waves analogous to spin waves and librational waves is proposed, and the corresponding quasi-particle characterizing elementary excitation of such motion is called a conformon.

The application of macroscopic magic-axis rotation to metallic copper is described. An order of magnitude improvement in precision has been obtained in Knight-shift determination for both copper isotopes. The accuracy of measurement of relative chemical shift for both isotopes in three cuprous halide reference compounds is likewise improved. At high speeds of rotation a residual rotationally-invariant linewidth is observed for both isotopes in metallic copper. This is attributed to the Ruderman-Kittel interaction, and enables this interaction to be determined for lighter metals for which it is ordinarily obscured by magnetic dipolar broadening.

Reference is made to the contribution of microscopic molecular motion within a solid to the effectiveness of macroscopic motion in securing $\mathrm{nmr}$ spectral narrowing, and examples of different cases are treated.
\end{abstract}

\section{INTRODUCTION}

The narrowing of the nmr spectra of solids by nuclear motion is a topic which goes back to the earliest days of $\mathrm{nmr}$. Motional narrowing in ice was discussed in the classic paper of Bloembergen, Purcell and Pound ${ }^{1}$, and was soon followed by a discussion of a number of other cases by Gutowsky and Pake $^{2}$ who provided a theoretical basis for the effect of specific types of 


\section{E. R. ANDREW}

molecular motion on the second moment of the nmr spectra. When supplemented by investigations of the spin-lattice relaxation time $T_{1}$, and of the rotating-frame relaxation time $T_{1 \rho}$, this type of investigation has provided a powerful means of studying the nature and kinetics of the motion of molecules, groups, ions and atoms in solids. A very large number of papers has been published over the past twenty years on a wide variety of solids, and this branch of the subject remains an active area of research.

In this paper I shall first discuss some recent investigations of this kind with which I have been associated, some of which show some novel features suggesting additional theory; then I shall report developments relating to a rather different form of molecular motion, conformational motion, which I first discussed in $1970^{3,4}$. After this I shall present some of our most recent work on motional narrowing of the $\mathrm{nmr}$ spectra of solids by macroscopic rotation of the specimen, especially mentioning an application to metals, and finally I shall mention the interrelation of macroscopic and microscopic motion.

\section{SOLID POLYMETHYLAMMONIUM CHLORIDES}

At the University of Florida, Mr. P. Canepa and I have been investigating the proton magnetic resonance spectra of the tetra-, tri-, di- and monomethylammonium ions in their polycrystalline chlorides, over a wide range of temperature. Full details will not be given here, but Figure 1 illustrates the motional narrowing of the pmr spectrum of tetramethylammonium chloride.

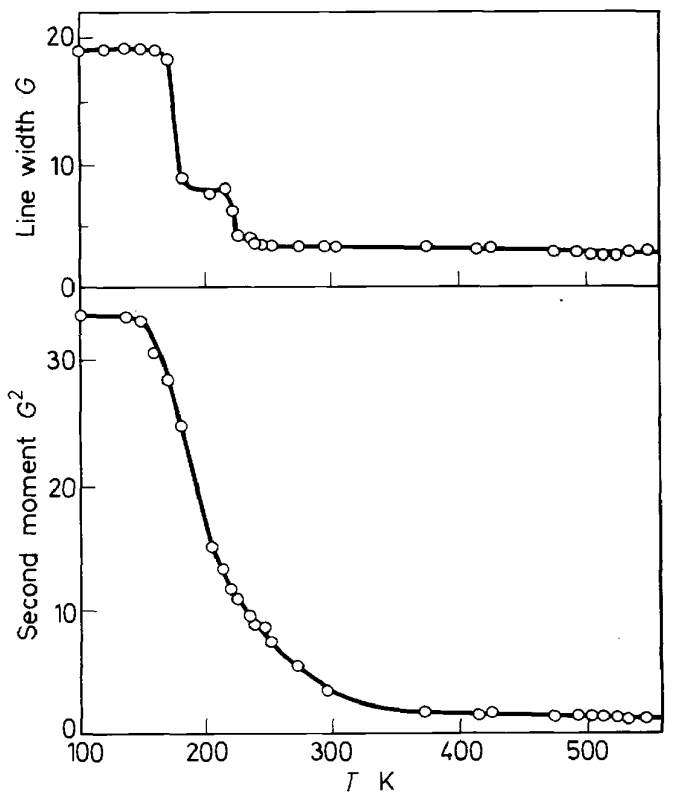

Figure 1. Motional narrowing of the pmr spectra of polycrystalline tetramethylammonium chloride. Upper diagram derivative linewidth; lower diagram second moment.

Abscissa: temperature (K)

Ordinate: upper diagram: linewidth (G);

lower diagram: second moment $\left(\mathrm{G}^{2}\right)$ 
The well-known rotational mobility of the methyl group about its triad axis is exhibited by all four compounds, though only in one of them (dimethylammonium chloride) is the restricting barrier low enough to enable spectral narrowing by tunnelling to be observed down to $4 \mathrm{~K}$. In the other three compounds the second moments of the spectra at the lowest temperatures agree well with values calculated ${ }^{5}$ on the assumption of a rigid array of protons. At higher temperatures the diversity of symmetry of the four ions is reflected in the interesting variety of motions of the whole ion. For tetramethylammonium chloride the second moment above $350 \mathrm{~K}, 1.4 \mathrm{G}^{2}$, agrees well with the value calculated on the assumption of isotropic or pseudoisotropic reorientation of the ions.

While it is likely that the potential barrier restricting the motion of the methyl groups may be lower than that restricting reorientation of the whole ion, so that the methyl group reorientation may be excited first as the temperature of tetramethylammonium chloride is raised, the second moment shows no intermediate plateau separating the effects of the two motions. It is possible that the slight arrest in the derivative linewidth at $8 \mathrm{G}$ may be associated with this. It will be noticed that the derivative linewidth narrows quite precipitately around $175 \mathrm{~K}$ over an interval of a few degrees. However this represents a relatively minor change in the shape of the spectrum to which the derivative is sensitive, and there is no corresponding rapid change in the second moment. It would clearly be inappropriate in such a case to use the relationship between linewidth and correlation time proposed by Bloembergen, Purcell and Pound ${ }^{1}$, and extended by Gutowsky and Pake ${ }^{2}$ to extract correlation times $\tau_{c}$ of the random nuclear motion at each temperature and attempt to obtain an activation energy $V_{0}$ from an Arrhenius plot. Such a procedure in the case of the extremely rapid narrowing of the derivative linewidth at $175 \mathrm{~K}$ would be grossly misleading, and would yield an activation energy much too high with a large error in the frequency factor. Waugh and Fedin ${ }^{6}$ had previously drawn attention to this problem, and showed that a better estimate of $V_{0}$ is to be obtained from the approximate relationship

$$
V_{0}=37 T_{c} \text { cal mole }{ }^{-1}
$$

where $T_{c}$ is the temperature at which the nmr spectrum narrows. For tetramethylammonium chloride this expression yields an activation energy of $6.5 \mathrm{kcal} \mathrm{mole}^{-1}$ for methyl group reorientation, and $8.2 \mathrm{kcal} \mathrm{mole}^{-1}$ for ionic reorientation.

More reliable values of activation energy are to be obtained from spinlattice relaxation measurements covering a wide range of temperature. $\mathrm{My}$ colleague, Mr. A. A. V. Gibson, has carried out such an investigation for solid tetramethylammonium chloride. Analysis of this work is still proceeding but preliminary values of activation energy are 5.5 and $9.3 \mathrm{kcal} \mathrm{mole}^{-1}$ for the two motions.

A more extreme situation is provided both by solid methylammonium chloride and by solid $l$-valine. In both cases an outer structure of the derivative spectrum weakens and finally peters out with increasing temperature while an inner structure strengthens and takes over. In these cases there is no continuous linewidth transition at all, and it is hazardous to assess a tempera- 


\section{E. R. ANDREW}

ture $T_{c}$ for use in estimating $V_{0}$ with the aid of equation 1 . In both cases however the observed second moment decreases smoothly with increase of temperature.

While a thorough study of the kinetics of molecular motion in solids requires a full investigation of nuclear spin-lattice relaxation it is a time-consuming exercise and requires specialized apparatus not available to all workers, who may often be content with an approximate estimate of $V_{0}$. As the previous examples have illustrated, the derivative linewidth frequently does not provide a reliable basis for estimating $V_{0}$. The second moment on the other hand is an experimental measure of the whole of the observed nmr spectrum. Its use in transition regions between plateau values has hitherto been supposed improper ${ }^{7,8}$ since strictly the second moment is invariant with respect to molecular motion, and measured values depend on the degree to which observations are carried into the wings of the spectrum. In the next section a development of theory is outlined which shows how the reducing second moment can nevertheless be justifiably used to describe the narrowing process and to extract an activation energy in suitable cases.

\section{SECOND MOMENT OF MOTIONALLY-NARROWED SPECTRA}

The Kubo-Tomita ${ }^{9}$ formulation of the motional narrowing of magnetic resonance spectra is taken as our starting point. Each nucleus interacts with a large number of neighbours, yielding a Gaussian spectral profile in absence of motion. When motion is present, characterized by a correlation time $\tau_{c}$, the lineshape around the centre is

$$
\begin{aligned}
I(\omega)=\left(\pi \omega_{1}\right)^{-1} \exp \left(\omega_{1} \tau_{c}\right)^{2} \sum_{n=0}^{\infty} \frac{(-1)^{n}}{n !} & \left(\omega_{1} \tau_{c}\right)^{2 n} \\
& \times \frac{\omega_{1} \tau_{c}+\left(n / \omega_{1} \tau_{c}\right)}{\left(\omega_{1} \tau_{c}+n / \omega_{1} \tau_{c}\right)^{2}+\left(\omega_{1} / \omega_{1}\right)^{2}},
\end{aligned}
$$

where $\omega_{1}^{2}$ is the second moment of the spectrum in absence of motion. Mrs. J. Lipofsky and I have computed $I(\omega)$ for a large number of values of the parameter $\omega_{1} \tau_{c}$ to give a family of spectral profiles in which the calculation is carried to $10^{-3} I(0)$. The lineshape changes progressively from Gaussian to Lorentzian.

If in the experimental situation measurements of the spectrum can only be carried to $\varepsilon I(0)$, where $\varepsilon$ is small and is determined by the signal-to-noise ratio and other instrumental factors, it is appropriate to compare the second moment of the experimental readings with theoretical second moment values in which the calculation is cut off at $\varepsilon I(0)$ also, ignoring the wings beyond that level. For non-zero values of $\varepsilon$ the second moment does then decrease steadily as $\tau_{c}$ decreases. Second moments have been computed from the family of computed spectral profiles for values of $\varepsilon$ ranging from 0.001 to 0.03 which covers all normal experimental situations, and the results are shown in Figure 2 for four values of $\varepsilon$. It will be noticed that the form of reduction is closely similar for all values of $\varepsilon$, and in extracting $\tau_{c}$ values from measured second 


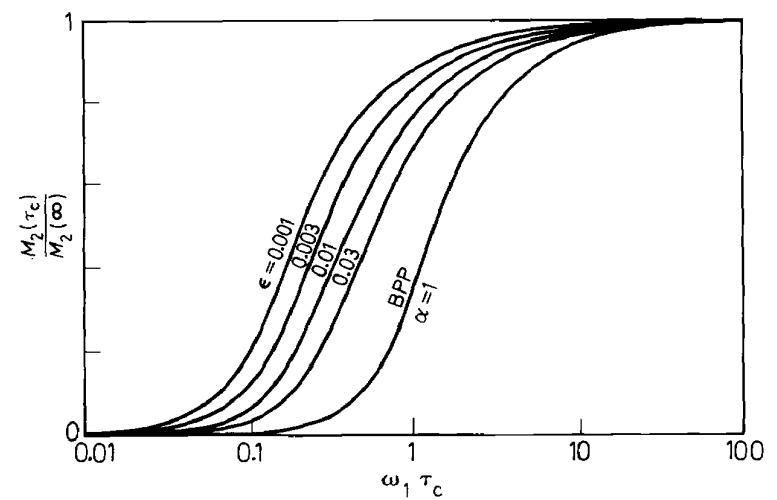

Figure 2. The calculated variation of second moment with correlation time. The family of four curves at the left are calculated from the theory of Kubo and Tomita ${ }^{9}$ as described in the text, for different values of the parameter $\varepsilon$. The curve to the right is based on equation 3 with $\alpha=1$.

Abscissa: $\omega_{1} \tau_{c}$

Ordinate: $M_{2}\left(\tau_{c}\right) / M_{2}(\infty)$

moments with the aid of these curves it is not therefore necessary to have an accurate estimate of $\varepsilon$ to obtain a reasonable value of $V_{\mathrm{o}}$.

Also plotted in Figure 2 is the relation

$$
\frac{M_{2}\left(\tau_{c}\right)}{M_{2}(\infty)}=\frac{2}{\pi} \tan ^{-1}\left(\alpha M_{2}^{\frac{1}{2}} \tau_{c}\right)
$$

based on the expression ${ }^{1,2}$ to which reference was made in Section 2. For convenience of plotting the parameter $\alpha$ has been taken as unity. Other values of $\alpha$ merely shift the curve along the logarithmic abscissa. This curve is rather similar to the family of curves we have obtained from the KuboTomita theory. The use of equation 3 should therefore lead to values of $V_{0}$ differing by not more than about $10 \%$ from values based on the family of curves obtained from the Kubo-Tomita theory.

\section{CONFORMATIONAL MOTION}

Many molecules are able to adopt more than one conformation in the liquid state and the rate of interconversion between conformers has frequently been studied by high-resolution $\mathrm{nmr}$ spectroscopy. In the solid state also it can be expected that such molecules may adopt more than one conformation ${ }^{3,4}$. It may be expected that the constraints imposed by crystalline packing will cause the barriers restricting interconversion to be higher than in the liquid state. Moreover conformers having equal energy in the liquid state may have different energy in the crystal on a given lattice site. Rapid interconversion between conformers narrows the nmr spectrum and provides a mechanism of nuclear spin-lattice relaxation. Calculations have shown ${ }^{3}$ that conformational motion is probably responsible for spectral narrowing in the lowtemperature phase of solid cyclobutane ${ }^{10}$. Conformational motion has also been demonstrated in solid perfluorocyclohexane ${ }^{11}$.

Solid cyclobutane exhibits a $\lambda$-type specific heat anomaly ${ }^{12}$ which is con- 


\section{E. R. ANDREW}

sistent with a conformational order-disorder process in the lower temperature phase. At the lowest temperatures the crystal would be ordered and the molecule would spend all its time in the 'right' conformation for its site. As the temperature is raised the proportion of time $p$ spent in the 'right' conformation would progressively fall below unity, and as a consequence of the cooperative disordering $p$ would reach the disordered value of $\frac{1}{2}$ at the transition temperature.

The spectrum of solid cyclobutane is dominated by the dipolar interactions between the close pairs of protons in the methylene groups. In the first analysis which Dr. Brookeman and I carried out ${ }^{3}$ we supposed the protonproton vectors to reorient rapidly between two equally probable orientations. The resulting reduced linewidth agreed well with experiment just below the $\lambda$ transition. Since disordering is almost complete here, the assumption that $p=\frac{1}{2}=1-p$ is reasonable. However at lower temperatures it must be assumed that $p$ lies between $\frac{1}{2}$ and 1 . I have therefore extended the calculations to general $p$ and have found how the linewidth varies with $p$ for each value of $2 \gamma$, the angle between the two orientations of the proton-proton vector. In the case of cyclobutane this is the dihedral angle of the molecule, which is close to $34^{\circ}$. Figure 3 shows the variation of linewidth $\delta \omega$ with $p$ for $2 \gamma=34^{\circ}$;

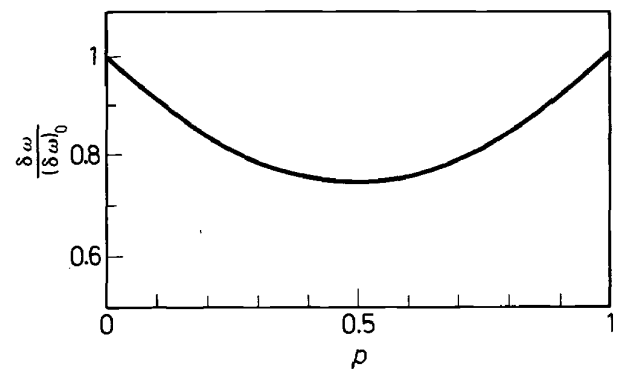

Figure 3. The variation of linewidth with conformational order parameter $p$.

there is a family of such curves for different values of $\gamma$. As one expects, the curve is symmetrical about $p=\frac{1}{2}$ since a crystal with a proportion $p$ of molecules in the 'right' conformation for their site is equivalent to one with a proportion $p$ in the 'wrong' conformation. Furthermore we notice that the minimum is a very shallow one, and consequently if the disordering is not quite complete just below the $\lambda$ temperature it makes little difference to the spectral narrowing expected. Indeed for values of $p$ between 0.4 and 0.6 the variation in linewidth is only $1 \%$.

Finally one may notice the analogy between 'right' and 'wrong' conformers on lattice sites in a partially ordered lattice and the situation in a partially ordered magnetic material. One may anticipate the propagation of disorder in a partially disordered crystal in a manner similar to spin waves. As in the related case of vibrational waves in solid hydrogen in which the quasiparticle describing elementary excitations is called a libron, the corresponding quasi-particle characterizing elementary excitation of conformational waves might be called a conformon. 


\section{MACROSCOPIC MOTION: APPLICATION TO COPPER}

It is now well-established that rapid rotation of solids narrows their $\mathrm{nmr}$ spectra. If the rotation axis is inclined at the magic angle, $54^{\circ} 44^{\prime}$, to the direction of the applied magnetic field, and the speed of rotation is fast enough, the anisotropic broadening interactions are largely removed from the spectrum. Fine structure arising from chemical shift differences or from spin multiplets may then be revealed as in the high-resolution spectra of fluids. A review of work in this field to 1970 has recently been published ${ }^{13}$. In this section the results are reported of an investigation in which Dr. J. L. Carolan, Mr. P. J. Randall and I have applied the technique to metals.

When a metal is rotated rapidly about the magic axis the central frequency of the $\mathrm{nmr}$ spectral line is determined by the isotropic Knight-shift $K=\frac{1}{3} \operatorname{tr} K$, where $K$ is the Knight-shift tensor. The narrower the line is rendered the more precisely its centre may be located, and the more accurately the isotropic Knight-shift may be determined. We have applied the method to copper at $25^{\circ} \mathrm{C}$ and have secured an order of magnitude improvement in precision in determining the Knight-shifts for ${ }^{63} \mathrm{Cu}$ and ${ }^{65} \mathrm{Cu}$. Solid cuprous halides $\mathrm{CuCl}, \mathrm{CuBr}, \mathrm{Cul}$ were used as reference materials. The specimens sealed in the turbine rotors were an intimate mixture of powdered copper metal and a cuprous halide powder, and they were spun at frequencies from 4 to $7 \mathrm{kHz}$. The mean experimental Knight-shifts obtained ${ }^{14,15}$ are shown in Table 1 . The error limits refer to a $99 \%$ confidence level. The differences in

Table 1. Knight-shifts for copper at $25^{\circ} \mathrm{C}$ (ppm)

\begin{tabular}{lrrr} 
& \multicolumn{3}{c}{ Reference material } \\
\cline { 2 - 4 } & \multicolumn{1}{c}{$\mathrm{CuCl}$} & $\mathrm{CuBr}$ & $\mathrm{CuI}$ \\
\hline${ }^{63} \mathrm{Cu}$ & $2338.3 \pm 0.8$ & $2392.9 \pm 1.2$ & $2335.5 \pm 1.1$ \\
${ }^{65} \mathrm{Cu}$ & $2340.8 \pm 1.1$ & $2395.1 \pm 1.6$ & $2337.4 \pm 1.9$ \\
\hline
\end{tabular}

chemical shift for the three halides underline the fact that absolute Knightshifts are not measurable and always involve the chemical shift of the reference compound. The results suggest a small isotope dependence of Knight-shift of about 2 ppm, marginally outside experimental error.

These results also provide accurate relative chemical shifts for both copper isotopes among the three halides. Relative to $\mathrm{CuCl}$ the chemical shifts (in ppm) of ${ }^{63} \mathrm{Cu}$ and ${ }^{65} \mathrm{Cu}$ in $\mathrm{CuBr}$ are respectively, $54.5 \pm 1.4$ and $54.2 \pm 1.9$, and in $\mathrm{CuI}$ are respectively $-2.8 \pm 1.4$ and $-3.4 \pm 2.3$. Thus there is no discernible isotope dependence of these chemical shifts. It is also to be noticed that there is no monotonic change in chemical shift with increasing halogen atomic number such as that observed in the solid caesium and rubidium halides ${ }^{16}$. However it should be noted that unlike the essentially ionic alkali halide crystals the cuprous halides are covalently bonded solids with a zinc blende structure at room temperature ${ }^{17}$.

For rotation frequencies above $4 \mathrm{kHz}$ the narrowed spectra of both copper 
isotopes in metallic copper reach limiting widths, which remain constant to $7 \mathrm{kHz}$, the highest rotation frequency used. Evidently this broadening arises from a rotationally invariant interaction, and is therefore most probably attributable to the Ruderman-Kittel indirect exchange coupling of the nuclear magnetic moments by the conduction electrons ${ }^{18,19}$. The larger residual linewidth and second moment of the less abundant ${ }^{65} \mathrm{Cu}$ isotope supports this interpretation. From the measured second moments of the residual spectra for the two copper isotopes we are in the process of extracting a value for the interaction constant $A_{i j} / h$ between nearest neighbour nuclei.

For a metal such as thallium, which has high atomic number and has two magnetic isotopes of substantial natural abundance, the nmr spectrum is dominated by the indirect nuclear coupling, whose contribution to the second moment exceeds that from the direct magnetic dipolar interaction by two orders of magnitude. In such cases the measurement of the indirect interaction presents no problem. However for a metal such as copper with lower atomic number the indirect interaction is much smaller and its contribution to the nmr second moment is masked by the dipolar interaction making it difficult to measure ${ }^{20}$. The removal of the dipolar broadening by high-speed magic-angle rotation, as in the present experiments with copper, thus provides a method of measuring the Ruderman-Kittel interaction even when it is small.

\section{MICROSCOPIC AND MACROSCOPIC MOTIONAL NARROWING}

In this section a combination of microscopic and macroscopic motions is considered. A question often asked, which is of some practical importance, is whether it is possible to narrow the nmr spectrum of a solid by high-speed magic axis rotation if the spectrum is already partially narrowed by microscopic molecular motion. Dr. Jasinski and I have considered this question ${ }^{21}$ and the answer turns out to be either yes, or no, or to some extent, according to the detailed circumstances of the specimen. We have approached the problem using the Kubo-Tomita formalism ${ }^{9}$, which enables a description to be given of the spectrum at all stages in the narrowing processes. The conclusions are summarized below.

Consider first a solid within which the molecules execute a restricted molecular motion which reduces the second moment (in angular frequency units) by an amount $\omega_{1 a}^{2}$ from a low-temperature rigid-lattice value of $\omega_{1}^{2}$ to a high temperature plateau value of $\omega_{1 b}^{2}$. This motion might for example be molecular reorientation about a single axis. Let the motion responsible for this reduction $\omega_{1 a}^{2}$ be characterized by a correlation time $\tau_{a}$, where $\omega_{1 a} \tau_{a}<1$ when the motion is effective in line narrowing. Any molecular motion capable of removing the residual second moment $\omega_{1 b}^{2}$, for example molecular diffusion, is characterized by a correlation time $\tau_{b}$, and since it is ineffective in linenarrowing it follows that $\omega_{1 b} \tau_{b}>1$. In these circumstances we have found ${ }^{21}$ that magic-angle specimen rotation at angular velocity $\omega_{r}$ reduces the linewidth further to

$$
\delta \omega \simeq \omega_{1 a}^{2} \tau_{a}+\left(\frac{\omega_{1 b}}{\omega_{r}}\right)^{2} \tau_{b}^{-1}
$$


The two terms arise from secular cross terms when the interaction Hamiltonian is averaged over both macroscopic and microscopic motions, and they reflect the spectral densities of the two motions characterized by $\tau_{a}$ and $\tau_{b}$, one fast and one slow, at frequencies $\omega_{r}$ and $2 \omega_{r}$.

The implications of equation 4 are best appreciated by consideration of some numerical values. Suppose the r.m.s. width $\omega_{1} / 2 \pi$ for the rigid lattice is $10 \mathrm{kHz}$, and this is reduced by the restricted molecular motion to a new plateau value $\omega_{1 b} / 2 \pi$ of $3 \mathrm{kHz}$, with $\tau_{a}=10^{-7} \mathrm{~s}\left(\omega_{1 a} \tau_{a} \ll 1\right)$. Suppose also that any random motion associated with the remaining width is slow, say $\tau_{b}=$ $10^{-2} s\left(\omega_{1 b} \tau_{b} \gg 1\right)$. Then if the specimen is rotated about the magic axis at say $6 \mathrm{kHz}$ the contributions to the linewidth of the two terms in equation 4 are respectively $60 \mathrm{~Hz}$ and $4 \mathrm{~Hz}$. Thus the line has been quite effectively narrowed by specimen rotation from $3 \mathrm{kHz}$ to about $64 \mathrm{~Hz}$. In this example the residual width arises mainly from the first term in equation 4 and is unaffected by the rotation. If the internal motion were faster, for example if $\tau_{a}=10^{-9} \mathrm{~s}$, then this source of residual broadening would become less important than the second term in equation 4. It is important to note therefore that the slow motion characterized by $\tau_{b}$ can also be a source of residual broadening. Suppose $\tau_{b}$ were somewhat shorter, $10^{-3} \mathrm{~s}$, which still satisfies $\omega_{1 b} \tau_{b}>1$; the residual linewidth from the second term is then $40 \mathrm{~Hz}$ which could seriously limit resolution. It is of course possible in principle to reduce this term further either by rotating the specimen faster, or by cooling it down so that $\tau_{b}$ becomes longer.

A quite different situation is presented by a moderately viscous fluid. Here there is isotropic reorientation and translation, so that $\omega_{1 b}=0, \omega_{1}=\omega_{1 a}$, and the residual linewidth of the specimen is given by the first term in equation 4 , namely $\omega_{1 a}^{2} \tau_{a}$ whether the specimen is at rest or is rotated. If $\omega_{r} \tau_{a} \ll 1$, frequencies $\omega_{r}, 2 \omega_{r}$ appear in the 'white' part of the spectrum of the molecular motion, and the residual width is independent of $\omega_{r}$ The molecular motions are not fast enough to narrow the line to the extent found in mobile liquids, but nevertheless they are fast compared with $\omega_{r}$ and it is physically reasonable that specimen rotation should have little effect. This case has also been discussed by Haeberlen and Waugh ${ }^{22}$, who considered isotropic motional narrowing for $\omega_{1 a} \tau_{a} \ll 1$.

So to summarize the situation we see that when the nmr spectrum of a solid is partially narrowed by restricted microscopic motion causing a reduced plateau value of the second moment, macroscopic rotation about the magic axis is capable of producing further substantial spectral narrowing. The limiting linewidth achieved depends on the values of the correlation times of the microscopic motions, and the line will be narrowest when the correlation time for the microscopic narrowing motion is very short, and when that for the rest of the broadening interactions is very large. In cases such as somewhat viscous liquids where there is a substantial microscopic isotropic narrowing, which does not reach a reduced plateau value of second moment, attainable speeds of macroscopic rotation will not further narrow the line. These conclusions do seem to be in agreement with most of the experimental facts ${ }^{13}$. 


\section{E. R. ANDREW}

\section{REFERENCES}

1 N. Bloembergen, E. M. Purcell and R. V. Pound, Phys. Rev., 73, 679 (1948).

2 H. S. Gutowsky and G. E. Pake, J. Chem. Phys., 18, 162 (1950).

3 E. R. Andrew and J. R. Brookeman, J. Mag. Res., 2, 259 (1970).

${ }^{4}$ E. R. Andrew, Physics Letters, 34A, 30 (1971).

5 J. H. Van Vleck, Phys. Rev., 74, 1168 (1948).

6 J. S. Waugh and E. I. Fedin, Soviet Physics-Solid State, 4, 1633 (1963).

7 A. Abragam, Principles of Nuclear Magnetism. Clarendon Press, Oxford (1961).

${ }^{8}$ E. R. Andrew and G. J. Jenks, Proc. Phys. Soc., 80, 663 (1962).

9 R. Kubo and K. Tomita, J. Phys. Soc. Japan, 9, 888 (1954).

10 M. J. R. Hoch and F. A. Rushworth, Proc. Phys. Soc., 83, 949 (1964).

11 J. D. Ellett, U. Haeberlen and J. S. Waugh, J. Am. Chem. Soc., 92, 411 (1970).

12 G. W. Rathjens and W. D. Gwinn, J. Am. Chem. Soc., 75, 5629 (1953).

13 E. R. Andrew, Progress in Nuclear Magnetic Resonance Spectroscopy, 8, 1 (1971).

14 E. R. Andrew, J. L. Carolan and P. J. Randall, Physics Letters 35A, 435 (1971).

15 E. R. Andrew, J. L. Carolan and P. J. Randall, Chemical Physics Letters 11, 298 (1971).

16 H. S. Gutowsky and B. R. McGarvey, J. Chem. Phys., 20, 1472 (1952).

17 A. F. Wells, Structural Inorganic Chemistry, Clarendon Press, Oxford (1962).

${ }_{18}$ M. A. Ruderman and C. Kittel, Phys. Rev., 96, 99 (1954).

19 N. Bloembergen and T. J. Rowland, Phys. Rev., 97, 1679 (1955).

${ }^{20}$ K. Tompa, Phys. Stat. Solidi, 18, 391 (1966).

21 E. R. Andrew and A. Jasinski, J. Phys. C, Solid St. Phys., 4, 391 (1971).

22 U. Haeberlen and J. S. Waugh, Phys. Rev., 185, 420 (1969). 